\title{
Utilização de plântulas de regeneração natural para restauração florestal
}

\author{
Using natural regeneration seedlings for forest restoration
}

\author{
Thomas Schröder, Frederico Dimas Fleig' \\ 'Universidade Federal de Santa Maria.
}

\section{Resumo}

A utilização de plântulas da regeneração de florestas naturais pode contribuir para a redução dos custos e ampla implementação de projetos de restauração de ambientes florestais. O objetivo deste estudo foi avaliar a sobrevivência de mudas de Canjerana obtidas da regeneração de florestas naturais para a produção de mudas de raiz nua e mudas de toco. A sobrevivência 60 dias após o plantio foi avaliada através de um Modelo Linear Generalizado com função de ligação logit, controlada pelo diâmetro da plântula e método de plantio. Mudas de raiz nua tiveram maior probabilidade de sobrevivência quando possuíam diâmetro de coleto inferior a um centímetro. Mudas de toco com mais de dois centímetros de diâmetro do coleto apresentaram probabilidade de sobrevivência maior que $80 \%$. A utilização de mudas de toco com mais de dois centímetros de diâmetro do coleto possuem grande capacidade de redução de custos em projetos de restauração de ambientes florestais.

Palavras Chave: Raiz nua; mudas de toco; sobrevivência; custos.

\begin{abstract}
Using regenerating seedlings from natural forests may contribute to cost reduction and widespread implementation of forest environment restoration projects. The aim of this study was to evaluate Cabralea canjerana obtained from natural regeneration seedling survival used to produce bare root and stump seedlings. Survival 60 days after planting was evaluated through a Generalized Linear Model with logit as link function, controlled by seedling diameter and planting method. Bare root seedlings had a greater survivorship probability when its diameter was smaller than one centimeter. Stump seedlings with more than two centimeters of diameter had a survival probability greater than $80 \%$. Stump seedlings with more than two centimeters may contribute to cost reduction in forest environment restoration projects.
\end{abstract}

Keywords: Bare root; stump seedlings; survival; costs. 


\section{INTRODUÇÃO}

A floresta atlântica possui hoje somente $12 \%$ da sua extensão original florestada (RIBEIRO et al., 2009). Atualmente existe um esforço no sentido de diminuir o dano humano através da restauração de florestas para proteger recursos naturais e a biodiversidade (LAMB; ERSKINE; PARROTTA, 2005). A principal barreira para a ampla implementação de projetos de restauração de ambientes florestais reside nos altos custos (RODRIGUES et al., 2009) de implantação e manutenção destas plantações. Mesmo em regiões onde existe a possibilidade de recuperação natural dos ecossistemas existe a necessidade de acelerar o processo de sucessão florestal.

Um dos principais custos da restauração do ambiente florestal reside na obtenção de mudas para implantação. Existem poucos viveiros florestais com grande capacidade instalada para produção de mudas de essências florestais nativas e, os que possuem tal capacidade não conseguem atender à demanda (Viveiro APREMAVI - comunicação pessoal). Além disso, existe uma pequena gama de espécies nativas que são atrativas no contexto sociológico, sendo estas espécies frutíferas (quase que exclusivamente da família Myrtaceae) em contextos mais urbanos e, espécies com madeira de valor comercial em contextos mais rurais.

A superação desta demanda de propágulos para a restauração do ambiente florestal pode ser conseguida através de técnicas com a utilização da regeneração natural de florestas naturais para a instalação de novas florestas (ADJERS et al., 1998; RIBEIRO et al., 2011; VIANI; BRANCALION; RODRIGUES, 2012). Esta técnica foi largamente utilizada para a implantação de plantios de Teca (Tectona grandis L. f.) na Ásia (GRIFFITH, 1939; BRASNETT, 1940; JACALNE; HEIMBAN; TADLE, 1957; KAOSA-ARD, 1982; JHA; CHOUDHARY, 1990), no entanto foi abandonada devido à maior sobrevivência $e$ controle genético de mudas produzidas em embalagens (DIXON et al., 1983; GROSSNICKLE, 2005, 2012). Esta técnica pode ser utilizada de duas formas, em ambas as mudas são obtidas do sub-bosque de florestas naturais e replantadas em novos espaços, estas mudas podem ser plantadas inteiras ou podem ter sua parte aérea totalmente removida. A primeira técnica se denomina plantio de mudas de raiz nua e a segunda técnica, plantio de mudas de toco (LAMPRECHT, 1990) (em inglês stump).

No entanto, para o sucesso desta técnica é necessário que a espécie utilizada tenha boa capacidade de brotação e certa resistência ao déficit hídrico. Neste contexto emerge a possibilidade de utilização da Canjerana (Cabralea canjerana Vell. Mart., Meliaceae) como uma espécie apropriada para tais fins. A Canjerana é uma espécie que ocorre ao longo de todo o domínio da Floresta Atlântica, suporta bem sombreamento tendo crescimento variável, podendo produzir até $14 \mathrm{~m}^{3} / \mathrm{ha}$ /ano (CARVALHO, 2002). A madeira possui boa durabilidade natural e agradável efeito estético (CARVALHO, 2002), possuindo bom valor monetário.

O objetivo deste trabalho foi avaliar a sobrevivência de plantio de mudas de canjerana obtidas a partir da regeneração natural transplantadas de duas formas, através de mudas de raiz nua e através do plantio de mudas de toco.

\section{METODOLOGIA}

\section{I. Descrição do local de estudo}

O presente estudo foi desenvolvido no município de Braço do Trombudo - SC, situado entre as coordenadas geográficas de $27^{\circ} 21^{\prime}$ de latitude sul e $49^{\circ} 52^{\prime}$ de longitude oeste, na região fisiográfica do Alto Vale do Itajaí. O clima é do tipo Cfa subtropical na classificação de Köppen, caracterizado como subtropical úmido sem estação seca definida. Os solos predominantes da região são cambissolos, formados por erosão eluvial (SANTA CATARINA, 1999, 2002). Os dados foram coletados no inverno de 2012 e são provenientes do primeiro desbaste de um povoamento com três anos de idade, instalado com espaçamento de 3 por 3 metros em curva de nível.

\section{Procedimento amostral}

Nos primeiros dias do mês de agosto de 2012 foram utilizadas 32 plântulas de Canjerana do sub-bosque de uma área de floresta natural próxima ao local do plantio. As mudas possuíam entre 0,7 e 2,7 centímetros de diâmetro de coleto. Mudas semelhantes foram pareadas para manter o experimento mais homogêneo o possível, metade destas plântulas foi truncada com um corte de $45^{\circ}$, 10 centímetros acima do coleto. Estas mudas foram plantadas em uma área de pastagem dominada por 
Paspalum spp .em um espaçamento de 1 x 1 metro mantendo o pareamento pré-estabelecido constituindo desta forma um Delineamento Inteiramente Casualizado (DIC). As mudas foram avaliadas quanto à sobrevivência 60 dias após o plantio.

\section{Procedimento estatístico}

Os dados foram analisados através de um Modelo Linear Generalizado no qual foram atribuídas variáveis dummy ao tratamento e á sobrevivência. Mudas de toco foram atribuídas o valor 1, mudas de raiz nua 0 , mudas vivas valor 1 e mudas mortas valor 0 . A sobrevivência foi considerada variável dependente com distribuição binomial, portanto foi utilizada uma função de ligação logit, para as variáveis independentes diâmetro do coleto, corte da parte aérea e a interação destas duas variáveis. Foram testados dois modelos, um com intercepto e outro sem intercepto, o melhor modelo foi escolhido com base no valor de AIC (Akaike Information Criteria).

A qualidade e robustez do modelo escolhido foram avaliadas através de um gráfico de resíduos ao longo da probabilidade de sobrevivência estimada. Para tanto os resíduos foram agrupados em dez partes para melhor visualização da variação entre média observada e estimada pontual. Os dados foram analisados através do pacote estatístico arm (GELMAN et al., 2012) disponível para interface do programa $\mathrm{R}$ (R Core Team, 2012).

\section{RESULTADOS E DISCUSSÃO}

As mudas plantadas por raiz nua apresentaram sobrevivência de $25 \pm 44 \%$ (média \pm desvio padrão) e as mudas de toco apresentaram sobrevivência de $50 \pm 52 \%, 60$ dias após o plantio. Estas médias não são significativamente diferentes entre si. No entanto, esta análise básica não permite inferências detalhadas sobre os diferentes métodos de plantio.

Dentre os Modelos Lineares Generalizados testados o melhor resultado foi encontrado com o modelo sem intercepto com valor de AIC igual a 39,8 , resulto ligeiramente melhor que o encontrado com o modelo com intercepto, 40,4. Na Tabela 1 são apresentados os valores das estimativas dos parâmetros para cada variável testada no modelo.

A análise gráfica do modelo desenvolvido e dos valores observados da Figura 1 permite uma análise mais aprofundada dos dados. Mudas de

Tabela 1. Estimativas dos parâmetros para predição da probabilidade de sobrevivência de mudas de Canjerana em função de seu diâmetro de coleto e método de plantio realizado em Santa Catarina

\begin{tabular}{ccc}
\hline Variável & Estimativa & Erro Padrão \\
\hline Diâmetro & $-0,9673$ & 0,4573 \\
Corte & $-4,0119$ & 2,5272 \\
Diâmetro* Corte & 3,5249 & 1,5220
\end{tabular}

Em que: Corte representa o truncamento da muda.

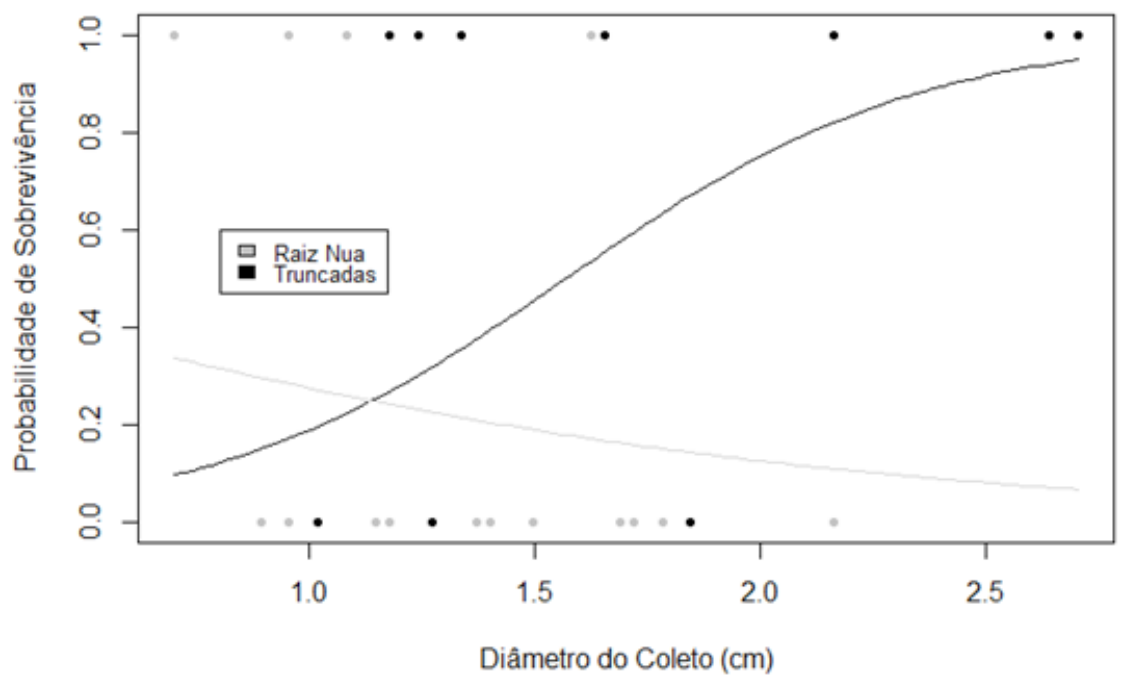

Figura 1. Probabilidade de sobrevivência estimada e observada em função de método de plantio e diâmetro de coleto de plântula de Canjerana em Santa Catarina. 


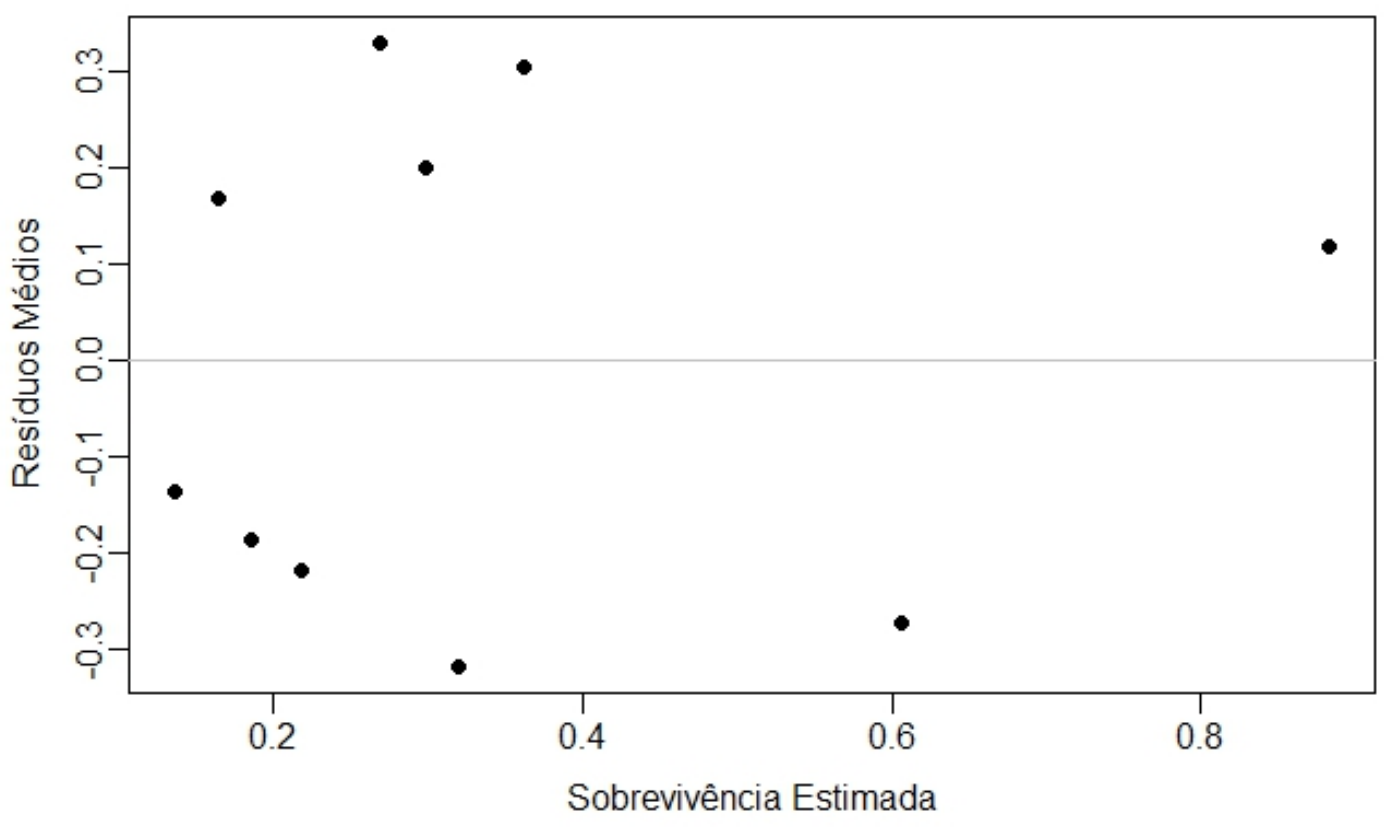

Figura 2. Resíduos agrupados médios da sobrevivência observada em função da sobrevivência estimada de mudas de Canjerana, através de um Modelo Linear Generalizado com função de ligação logit, para Santa Catarina.

raiz nua possuem probabilidades de sobrevivência maiores que mudas de toco quando estas possuem até um centímetro de diâmetro, no entanto esta probabilidade pode ser considerada baixa, em torno de $30 \%$. A partir de um centímetro de diâmetro mudas de toco possuem maior probabilidade de sobrevivência. Mudas com mais de dois centímetros de diâmetro de coleto, e de toco possuem probabilidade de sobrevivência maiores que $80 \%$, sendo ideais para utilização em projetos de restauração florestal.

A estabilidade do modelo ao longo da amplitude da estimativa pode ser visualizada na Figura 2, que representa os resíduos agrupados da estimativa. Estes resíduos encontram-se distribuídos de forma homogênea, não apresentando nenhuma tendência de superestimativa ou subestimativa da probabilidade de sobrevivência.

Em um estudo semelhante em que se utilizou da regeneração natural de florestas para produção de mudas (VIANI; BRANCALION; RODRIGUES, 2012), verificou-se que existe a possibilidade de armazenamento destas plântulas por até um dia sem danos significativos à sobrevivência destas no plantio. Outra técnica passível de utilização neste contexto de restauração florestal, que foi testado em um experimento semelhante pelo autor é o encharcamento do solo por ocasião do plantio, o que retira ar do solo e aumenta o contato das raízes com o mesmo, evitando perdas adicionais de água pela planta.

\section{CONCLUSÕES}

A utilização de plântulas da regeneração de florestas naturais para produção de mudas permite uma redução dos custos e maior disponibilidade de mudas para restauração de ambientes florestais;

Mudas de raiz nua devem ter menos de um centímetro de diâmetro no coleto para terem maior probabilidade de sobrevivência que mudas de toco;

Mudas de toco com mais de dois centímetros de diâmetro do coleto possuem probabilidade de sobrevivência maior que $80 \%$

\section{REFERÊNCIAS BIBLIOGRÁFICAS}

ADJERS, G. et al. Production of planting stock from wildings of four Shorea species. New Forests, Purdue, v. 16, n. 3, p. 185-197, 1998.

BRASNETT, N. Stump planting. East African Agricultural Journal, Nairóbi, v. 5, n. 1, p. 387-388, 1940.

CARVALHO, P. E. R. Canjarana. Colombo: Embrapa Florestas, 2002. 17 p. 
DIXON, R. et al. Comparative water relations of container-grown and bare-root ectomycorrhizal and nonmycorrhizal Quercus velutina seedlings. Canadian Journal of Botany, Young House, v. 61, n. 6, p. 1559-1565, 1983.

GELMAN, A. et al. arm: Data Analysis Using Regression and multilevel/Hierarchical Models. New York, 2012. Versão 1.5-08. Sistema Operacional Windows 64x.

GRIFFITH, A. An investigation into the best age and diameter of stump to use when stump planting teak (Tectona grandis) in areas having a general west coast type of climate. Indian Forest Service Silvica, Calcutá, v. 3, n. 5, p. 165-194, 1939.

GROSSNICKLE, S. C. Importance of root growth in overcoming planting stress. New Forests, Purdue, v. 30, n. 2-3, p. 273-294, 2005.

GROSSNICKLE, S. C. Why seedlings survive: influence of plant attributes. New Forests, Purdue, v. 43, n. 5-6, p. 711-738, 2012.

JACALNE, D. et al. A study of the stump planting of mahogany (Swietenia macrophylla King). Philippine Journal of Forestry, Manila, v. 13, n. 2, p. 63-80, 1957.

JHA, M.; CHOUDHARY, L. Trial on stump planting of some tree species. Indian Forester, Bombain, v. 116, n. 4 , p. 283-285, 1990.

LAMB, D. et al. Restoration of degraded tropical forest landscapes. Science, Washington, v. 310, n. 1, p. 1628-1632, 2005.

LAMPRECHT, H. Silvicultura nos Trópicos-Ecossistemas florestais e respectivas espécies arbóreas-Possibilidade de aproveitamento sustentável. Göttingen: Eschborn, 1990. 343 p.

R Core Team. R: A language and environment for statistical computing.Vienna,2012. Versão 2.15.1. Sistema Operacional Windows 64x.

RIBEIRO, M. C. et al. The Brazilian Atlantic Forest: How much is left, and how is the remaining forest distributed? Implications for conservation. Biological Conservation, Amsterdã, v. 142, n. 6, p. 1141-1153, 2009.

RIBEIRO, T. M. et al. Sobrevivância e crescimento inicial de plântulas de Euterpe edulis Mart. transplantadas para clareiras e sub-bosque em Floresta Estacional Semidecidual, em Viçosa, MG. Revista Árvore, Viçosa, v. 35, n. 6, p. 1219-1226, 2011.

RODRIGUES, R. R. et al. On the restoration of high diversity forests: 30 years of experience in the Brazilian Atlantic Forest. Biological Conservation, Amsterdã, v. 142, n. 6, p. 1242-1251, 2009.

SANTA CATARINA. Zoneamento agroecológico e socioeconômico do Estado de Santa Catarina. EPAGRI - Empresa de Pesquisa Agropecuária e Extensão Rural de Santa Catarina S.A., 1999. 362 p.

SANTA CATARINA. Mapa de Solos - Unidade de Planejamento Regional Alto Vale do Itajaí. . EPAGRI - Empresa de Pesquisa Agropecuária e Extensão Rural de Santa Catarina S.A., 2002. 1 p.

VIANI, R. A. G. et al. Corte foliar e tempo de transplantio para o uso de plântulas do sub-bosque na restauração florestal. Revista Árvore, Curitiba, v. 36, n. 2, p. 331-339, 2012. 